\title{
Die Zukunft der Kinderradiologie - Ein Strategiepapier
}

Die Gesellschaft für Pädiatrische Radiologie e. V. (GPR) hat ein Strategiepapier zur Entwicklung der Kinder- und Jugendradiologie veröffentlicht (Fortschr. Röntgenstr. 2020; DOI 10.1055/a-11032339). Prof. Dr. Hans-Joachim Mentzel (Jena), Präsident der GPR, ist gemeinsam mit einer Reihe führender Kinderradiologen und Radiologen Autor des Papiers. Hier skizziert er Beweggründe und die Zielrichtung des Papiers.

\section{Was war der Impuls für das Strategie- papier?}

Wir formulieren das bereits in der Präambel des Papers: Gemäß UN-MenschenrechtsCharta haben Kinder das Anrecht auf das erreichbare Höchstmaß an Gesundheit. Für uns Kinderadiologinnen und Kinderradiologen schließt das die bildgebende Diagnostik durch unsere Fachvertreter ein. Die bestmögliche bildgebende Diagnostik dieser Patientengruppe kann nur durch speziell ausgebildete Ärzte, Kinderadiologinnen und Kinderradiologen erfolgen.

\section{Sie sehen aktuell diese Versorgungssitua- tion gefährdet?}

Ja. Die gemäß den Konventionen erforderliche Versorgungssituation durch Kinderund Jugendradiologen ist aktuell flächendeckend nicht gegeben. Das wird sich in Zukunft ohne aktives Gegensteuern auch weiter verschärfen.

\section{Wen und Was wollen Sie mit dem Strate- giepapier erreichen?}

Wir adressieren in diesem Papier eine Reihe von Partnern. Unsere Kollegen aus Radiologie und Neuroradiologie, mit denen wir über Modelle zur gemeinsamen Weiterbildung und Forschung sprechen möchten. Unsere Vorschläge und Forderungen richten sich aber vorrangig an die Politik und die Seite der Kostenträger, damit zum Beispiel der unzureichend finanzierte Bereich ambulant-kinderradiologischer Leistungen Aufwertung erfährt.

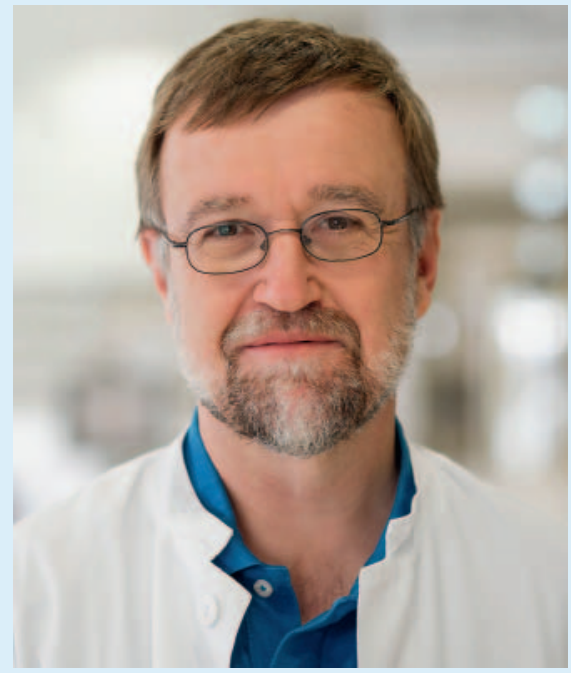

Prof. Dr. Hans-Joachim Mentzel

Der Vorstand der GPR wird daher die in diesem Papier formulierten Thesen in alle relevanten Gremien der Politik tragen und für eine Verbesserung der Situation des Faches in der Krankenversorgung und in der Forschung kämpfen. Dazu rufen wir auch jedes einzelne Mitglied unserer eigenen Fachgesellschaft auf. Und wir sprechen mit diesem Papier den radiologischen Nachwuchs an. Das Fach Kinder- und Jugendradiologie vereint die Innovationskraft der bildgebenden Medizin mit allen Herausforderungen und beglückenden Momenten der Kinder- und Jugendmedizin. Unser Papier ist auch ein Statement: Wir sind ein hochinteressantes Fachgebiet mit guten Entwicklungsmöglichkeiten. Es lohnt sich, hier seine Berufung zu finden!

Entwicklung der Kinder- und Jugendradiologie - Strategiepapier des Vorstands der Gesellschaft für Pädiatrische Radiologie:

\section{https://www.thieme-connect.de/ products/ejournals/html/10.1055/ a-1103-2339}

\section{ÜBER DIE GESELLSCHAFT FÜR} PÄDIATRISCHE RADIOLOGIE E. V.

Die GPR ist die wissenschaftliche Fachvertretung für alle in Deutschland, Österreich und der Schweiz tätigen Jugend- und Kinderradiologinnen und -Radiologen. 1963 gegründet, zählt die Gesellschaft aktuell über 345 Mitglieder. Ziel ist es, alle bildgebenden Verfahren nach dem neuesten wissenschaftlichen Stand für Kinder und Jugendliche jeglichen Alters einzusetzen, für ihre stete Weiterentwicklung zu sorgen, insbesondere unter Beachtung des Strahlenschutzes. Die GPR ist aktuell an mehr als 30 Leitlinien beteiligt.

www.kinder-radiologie.org 